\title{
La fotografía como medio para la interpretación sonora
}

\author{
Photography as a means for sound interpretation
}

Irene Guirado Isla

Universidad de Málaga, España.

iregisla93@gmail.com

Recibido 14/03/2018

Aceptado 27/05/2018
Revisado 27/05/2018

Publicado 01/07/2018

\section{Resumen}

El presente artículo busca responder al interrogante sobre si sería posible el uso de productos fotográficos para la interpretación sonora al piano. Para ello, realizo una investigación artística personal en la que pretendo encontrar las herramientas de observación en la imagen para traducirlas a una exposición auditiva. Como fuente de conocimiento para ello, parto de las partituras de grafismos musicales (Schröder, según citado en Buj, 2013) cuya forma sonora nace de la interpretación de los elementos visuales. De este modo, tomo dos fotografías tomadas en la costa malagueña para su posterior interpretación visual en función a un posible producto sonoro.

\section{Abstract}

This article is a research about the use of photos as a score to the piano interpretation. For this, I do a artistic research which I expect to find the analytic tools for It makes possible see sounds in the image. As knowledge source for this, I have based on graphic notation (Schörder, aforementioned in Buj, 2013) whose sound form it based on the visuals elements interpretation. In this way, I've chosen two photographies taked in the Malaga coast for a last visual interpretation in according to their possibles sounds. 
Revista de Estudios en Sociedad,

Artes y Gestión Cultural

Número 14

Julio 2018

ISSN: 2340-9096

DOI: https://dx.doi.org/10.17561/rtc.n14.8 www.terciocrecientecom

http://revistaselectronicas.ujaen.es/index.php/RTC
Investigación

Palabras clave / Keywords

Notación gráfica, grafismo musical, investigación artística, experimento sonoro.

graphic notation, music graphism, artistic research, experiment about sound.

Para citar este artículo

Guirado Isla, Irene (2018). La fotografía como medio para la interpretación sonora. Tercio Creciente, 14, págs. 97-108. https://dx.doi. org/10.17561/rtc.n14.8 


\section{1.- Inquietudes}

Este A raíz de una sesión acerca de la educación artística junto al profesor Fernando Hernández (2017), me sentí interesada por sistemas de notación musical más libres en cuanto al valor otorgado a cada símbolo. De esta forma, y apoyándome en los conocimientos de notación gráfica y grafismo musical existentes (Schröder, según citado en Buj, 2013), determiné que llevaría a cabo una investigación artística para conocer si es posible el uso de una fotografía como fuente de notación gráfica para una interpretación sonora al piano. Mi interés surge, principalmente, para aumentar los rangos de visión y aprendizaje en la interpretación pianística, no dejando con ello a un lado la partitura tradicional, sino aumentando las herramientas mediante el descubrimiento y la investigación en nuevos métodos de lectura sonora. Igualmente, la búsqueda de este tipo de lecturas musicales corresponde a una inquietud personal de libertad tanto en la lectura como en el resultado, queriendo de este modo también hallar caminos en la interpretación musical cuyos productos sean, en cierta manera, desconocidos al comienzo de la misma; tal y como buscaba Cage mediante la indeterminación (Íbidem).

Para cumplir con lo propuesto, propongo la lectura visual de dos fotografías atendiendo a la disposición, el color, las formas y el movimiento propio de aquello que representa, así como el movimiento propio que muestra la fotografía. Esta primera lectura será pasada a un producto sonoro atendiendo a cuatro caminos diferentes abarcando desde una visión más tradicional hasta una experimentación sonora (Cage, 2012).

En conclusión, las preguntas que me acompañan durante el proceso de la presente investigación artística son: ¿Puede una fotografía funcionar tal como una partitura para el/la intérprete? ¿Puedo descubrir y conocer mejor la imagen de la fotografía mediante la interpretación musical?

\section{Recogida de datos: la interpretación sonora desde lo visual}

La lectura de una fotografía para una posterior traducción al lenguaje musical se apoya en la tendencia propia del siglo XX de uso de elementos visuales para la interpretación musical-sonora, la cual se desarrolló como respuesta a la necesidad de expresar en las partituras ideas no pensadas anteriormente en la composición musical (García, 2007) y, por consecuente, sin símbolos en la notación tradicional. Igualmente, y como indica Buj (2013), el hecho de querer buscar nuevas formas de notación musical es el resultado de "(...) un deseo de apertura, creatividad y libertad por parte de los compositores" (p. 6).

Dentro de la notación gráfica, existieron según Schröder (citada en Buj, 2013) principalmente dos caminos: 1) la notación gráfica, que consiste en la realización de símbolos visuales dentro de las partituras que corresponden a una lectura sonora según el o la intérprete considere o el autor indique, como es el caso de las lecciones de entonación del compositor malagueño Ramón Roldán (García; Iglesias; Iglesias; Robles \& Roldán, s.f.). El otro camino, y el más relacionado con el propósito de que una fotografía funcione a modo de partitura, especificado también por Schröder (citada en Buj, 2013) es 2) el grafismo musical, el cual el concepto de partitura 
cambia radicalmente siendo un objeto visual la notación gráfica en sí misma, siendo totalmente tarea del/de la intérprete determinar qué producción sonora corresponde a la imagen. Un ejemplo de grafismo musical es December 1952 de Earle Brown y, precisamente por la naturaleza libre y podríamos decir personal de este tipo de lectura musical es, como afirma Buj (Íbidem) muy complicado que se produzcan dos interpretaciones iguales, siendo por tanto una ardua tarea identificar la obra de forma sonora únicamente. La investigación artística aquí presentada se basaría, por tanto, en este último camino, aunque de igual modo se determinará en uno de los procesos propuestos en la investigación, una lectura de la partitura semejante a la notación gráfica.

John Cage también fue uno de los autores que incorporó gráficos a su notación musical, tal y como se observa en algunas de sus obras de azar de los años 50, como 50 1/2 (García 2007). De hecho, la conocida obra del citado autor 4'33"se inspira en White Paintings del autor Robert Rauschenberg (Buj, 2013). Los caminos tomados por John Cage en la notación gráfica en sus piezas surgen por la tendencia compositiva de la indeterminación, la cual para John Cage suponía

\section{(...) provocar una situación en la cual} pueden pasar cosas que no están bajo mi control...Un ejemplo de indeterminación es cualquiera de las piezas de la serie Variations, que parecen cámaras que no te dicen qué foto hacer pero te permiten hacer una foto..." (John Cage citado en Feisst, 2002, según citado en Buj, 2013, p. 8)

Como hemos observado, existiría una estrecha relación entre el grafismo musical(Schröder, según citado en Buj, 2013) y el objetivo de crear arte sonoro a través de una fotografía, ya que consistiría en llevar al lenguaje musical elementos de la conjugación visual. No obstante, también resulta necesario mencionar la relación existente entre la idea de tomar una fotografía y llevarla a la producción sonora, con la creación musical de tipo programática (Fubini, 2007) puesto que, este estilo, tomaba elementos extramusicales y, ya fuese mediante la imitación de los sonidos propios de los mismos o a través de la evocación, se llevaban al lenguaje musical (Ortega, 2008). De este modo, por ejemplo, encontramos obras musicales como la Suite Española de Albéniz (ed. por Lechner, 1996) que recoge su percepción de diversas provincias españolas en escritos musicales o la pieza Wasserklavier de Luciano Berio (según citado en Gaspar, 2016), por la cual se busca organizar los sonidos de tal modo que resulten semejantes a los propios del agua. Sin embargo, aunque, como en el caso anterior, se pretenda hacer alusión a un elemento externo al lenguaje musical, en general el autor o la autora no pretende que sea una fiel imitación al sonido real porque, como indicaba John Paynter (según citado en Ortega, 2008), la obra musical no consiste en transformar las experiencias o producciones de otros lenguajes artísticos a un concepto sonoro que transmita con precisión la misma idea o imagen. Este último hecho en nuestro caso discerniría en los caminos propuestos para la lectura de la fotografía como medio para la creación sonora, a excepción del primero, pues en el caso de la música programática debemos recordar que el elemento extramusical funcionaba en términos generales como fuente de inspiración para la labor compositiva (Ortega, 2008), mientras que la fotografía en mi propuesta busca ser una notación musical.

\section{La investigación: procesos y resultados}

\subsection{Cuestión metodológica}

Tomando las premisas especificadas con anterioridad, el principal objeto de la presente investigación es: ¿Cómo puedo observar una fotografía para obtener, interiormente, una respuesta sonora?

Para dar respuesta a este interrogante me baso en una investigación artística desarrollada mediante mi propia experiencia en el proceso de la misma ya que, como indica Siri Hustvedt:

(...) ¿quién es el yo de la página? ¿Por qué usarlo? (...) Pero quiero implicarme en él. No deseo esconderme detrás de un trabajo 
académico porque, al recurrir a mi experiencia subjetiva, puedo, y creo que consigo, iluminar los problemas que pretendo desentrañar." (Hustvedt, 2013, p. 11 según citado en Espíritu, 2014, p. 62)

\subsection{Procedimientos a seguir}

Los procedimientos que planteo para la interpretación sonora de las fotografías son 4:

1) Camino tradicional: En este camino la fotografía no se leería, en su lugar se plantea como objeto de inspiración estética, tal y como se ha descrito anteriormente que sería su función en la música programática. De este modo, se buscará que la figuración o melodía elegida mantenga algún tipo de relación con los elementos de la fotografía, pero ello sería desarrollado junto a otros rasgos más personales (Ortega, 2008).

2) Camino de traducción: Planteamiento semejante al anterior pero con una presencia más protagonista de los elementos de la fotografía. En este caso, se tratará de identificar aspectos de la fotografía con determinados sonidos y/o movimientos al piano, realizándose así una lectura de la imagen mediante la identificación de dichos elementos para la interpretación sonora. Igualmente, se ve influenciado por la tradición en aquellos elementos con un movimiento o sonido poco identificable como es el caso, por ejemplo, del cielo. En el cielo, por continuar con el mismo ejemplo, el hecho de que sea de día se relacionaría (por mi experiencia propia) con un registro claro y un movimiento sonoro tranquilo.

3) Camino de asociación: En esta opción de lectura de fotografía, se observarán las formas de los elementos visuales más destacables de la imagen para identificarlos con un movimiento semejante en el piano, en el caso de las formas, o con un tipo de configuración de sonidos, en el caso de los colores. Este camino podría a priori resultar muy parecido al anterior, sin embargo la diferencia entre ambos radica en que el camino de traducción busca hallar elementos para relacionarlos con un movimiento o sonido al piano que haga alusión al propio de dicho aspecto en la experiencia diaria. Mientras tanto, el camino propuesto en este apartado tiene como objetivo identificar al elemento de la foto libre del sonido que le acompaña en la experiencia del día a día, asignándole así una interpretación sonora en el piano según su lectura musical dentro de la fotografía. No obstante, evidentemente siempre existirá una influencia en la lectura visual de mi experiencia propia, por lo que algunos elementos que identifique en la fotografía estarán interpretados según mi relación en la vida con los mismos.

4) Camino de simbolización: En este camino nuevamente se identificarán elementos de la imagen pero, en esta opción, para alejarnos de cualquier experiencia personal o influencia sociocultural, se le asignarán al azar movimientos y usos técnicos del piano, de los cuales, he escogido los siguientes:

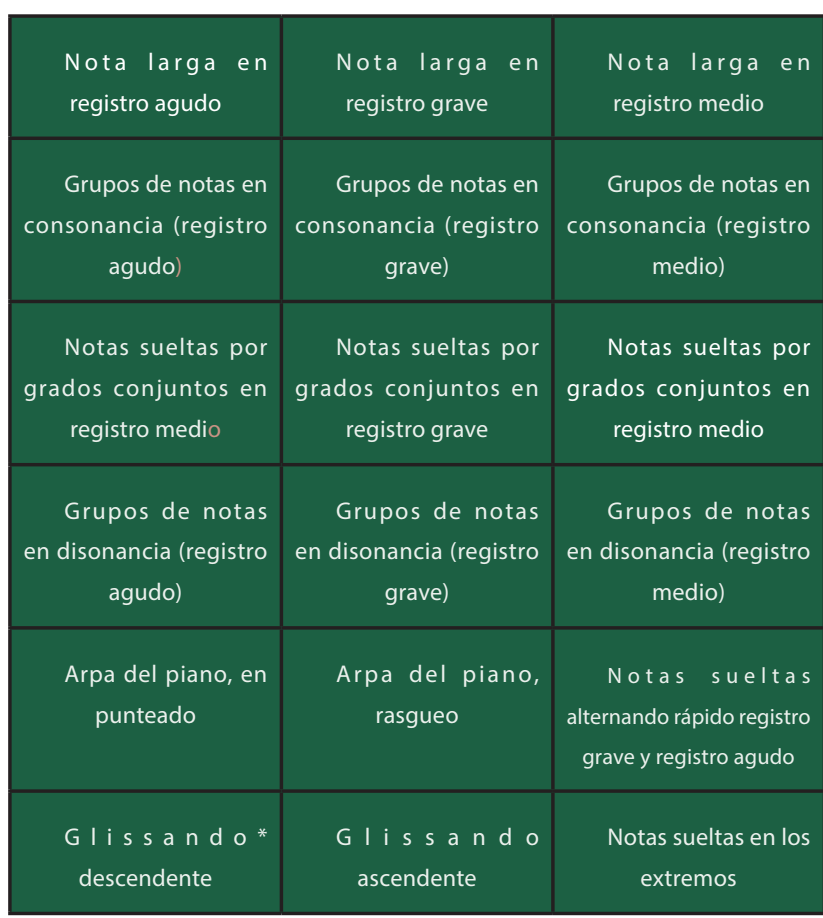




\subsection{Desarrollo de la investigación}

\subsubsection{Las fotografías}

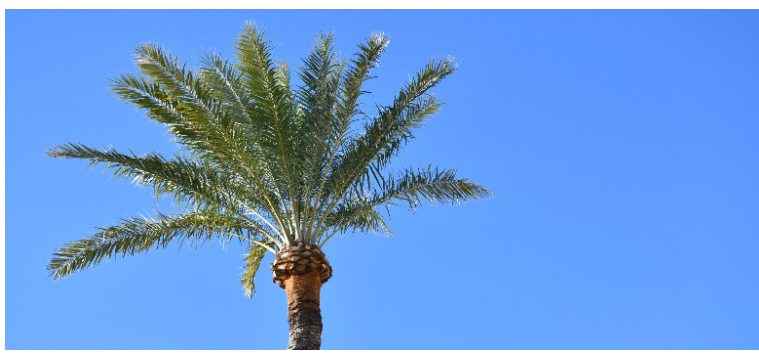

Fotografía 1 Fotografía de: Arcas Isla, 2018.

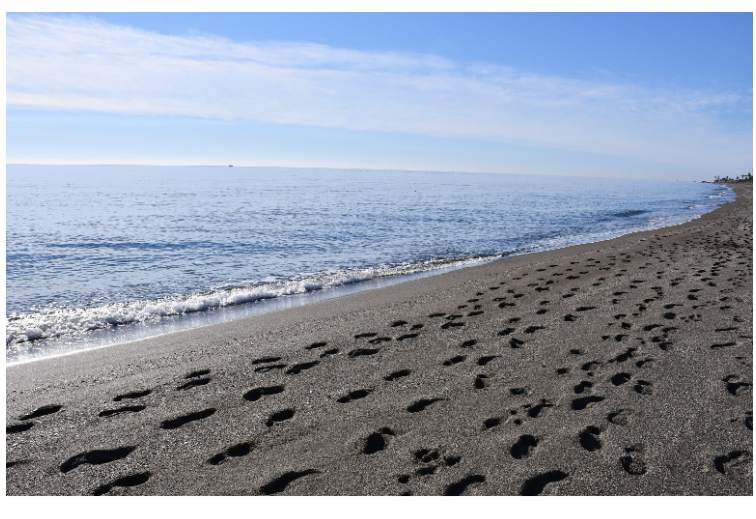

Fotografía 2 Fotografía de: Arcas Isla, 2018.

\subsubsection{Toma de decisiones en la investigación}

\section{Fotografía 1}

\section{- Camino tradicional}

Al investigar la fotografía bajo este enfoque tuvo como resultado una composición sencilla de tipo tonal, con forma ABA en Fa\# menor. La tonalidad fue elegida debido a mi relación personal del mar con la luna, la cual se relaciona a su vez con la nota de
Fa\# según los estudios del movimiento de la luna expuestos en la web Planetware (Dobretzberger -responsable-, s.f.). La parte $B$ está en el relativo mayor de la tonalidad principal, La mayor, y este cambio dentro de la pieza se debe a las huellas que se observan en ella, que me invitan a pensar en el paso de la vida. Por otro lado, el tipo de movimiento de las manos que presentan los patrones rítmicos principales de la pieza pretenden hacer alusión al movimiento circular propio de los movimientos del mar.

\section{- Camino de traducción}

En esta parte de la investigación busqué la forma de semejar los sonidos que transmiten los elementos de la fotografía con sonidos que pudiesen ser producidos con el piano. La lectura de esta fotografía la propongo semejante a como se realizaría una lectura de texto en mi país (España), siendo ésta de izquierda a derecha y en dirección descendente. De este modo, la fotografía queda dividida por sus elementos, en tres fragmentos: cielo, mar y arena. El cielo, queda reflejado mediante el uso de sonidos agudos, concebidos culturalmente como "claros". Para el mar, hago uso del arpa del piano, actuando sobre ella directamente desde el interior del piano mediante el rasgado, haciendo movimiento ascendente y descendente. El inicio de la pieza es mediante las notas Sol, Do y Si que corresponderían a las letras $M, A$ y $R$ respectivamente, lo cual uso como título introductorio en la pieza. Este alfabeto pasado a sonidos lo realizo desde hace años y fui inspirada a hacerlo por el Motivo B A C H del compositor J. S. Bach el cual, a su vez, inspiró a Schoenberg (May, 1993) (para ver mi alfabeto, consultar apartado 5 , notas).

Finalmente, en la arena el objeto visual que queda más destacado son las huellas $y$, por ello, uso el registro grave del piano, por la profundidad, y toco diversas notas sueltas, ya que las pisadas son separadas entre sí. 


\section{- Camino de asociación}

Para este enfoque, realicé un pequeño análisis acerca de los colores y formas de la fotografía, asociándolos a un movimiento y registro propio del piano según los conocimientos socioculturales predominantes: colores pastel con notas agudas, colores oscuros con notas graves, etc. En cuanto a los movimientos observados en los elementos visuales, busqué en el piano movimientos semejantes. Al igual que en el caso anterior, la lectura de la fotografía para la interpretación pianística se realiza igual que la lectura de un texto según cómo se hace en mi país.

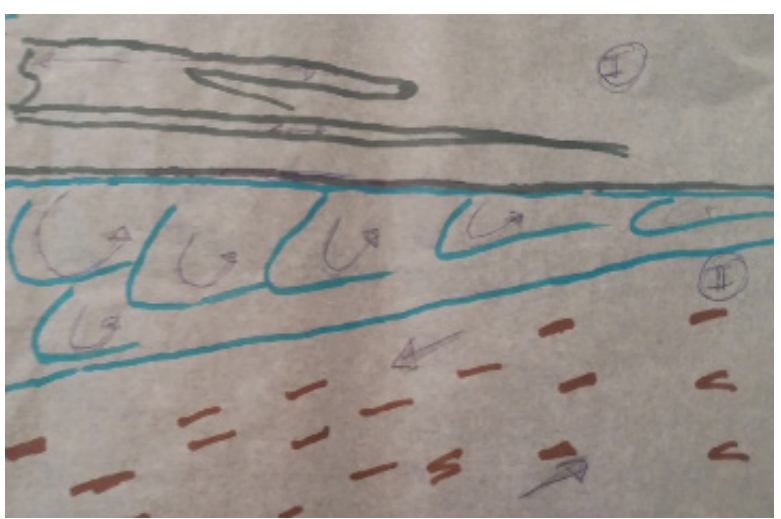

¿Qué veo en la imagen sonoramente?

\section{- Camino de simbolización ${ }^{1}$}

En este caso, siguiendo el procedimiento propuesto, en mi análisis de la imagen concluí los siguientes aspectos, de ascendente a descendente en la fotografía: difuminado blanco, fondo azul, línea color blanco, fondo azul color más claro, líneas en el mar, rompeolas, fondo marrón y huellas en la arena. Posteriormente, a cada uno de ellos le asigné una de las técnicas mencionadas en la tabla del apartado 3.2. del presente artículo, mediante el azar. De este modo, como resultado, quedó la siguiente simbolización de los elementos para la interpretación pianística:

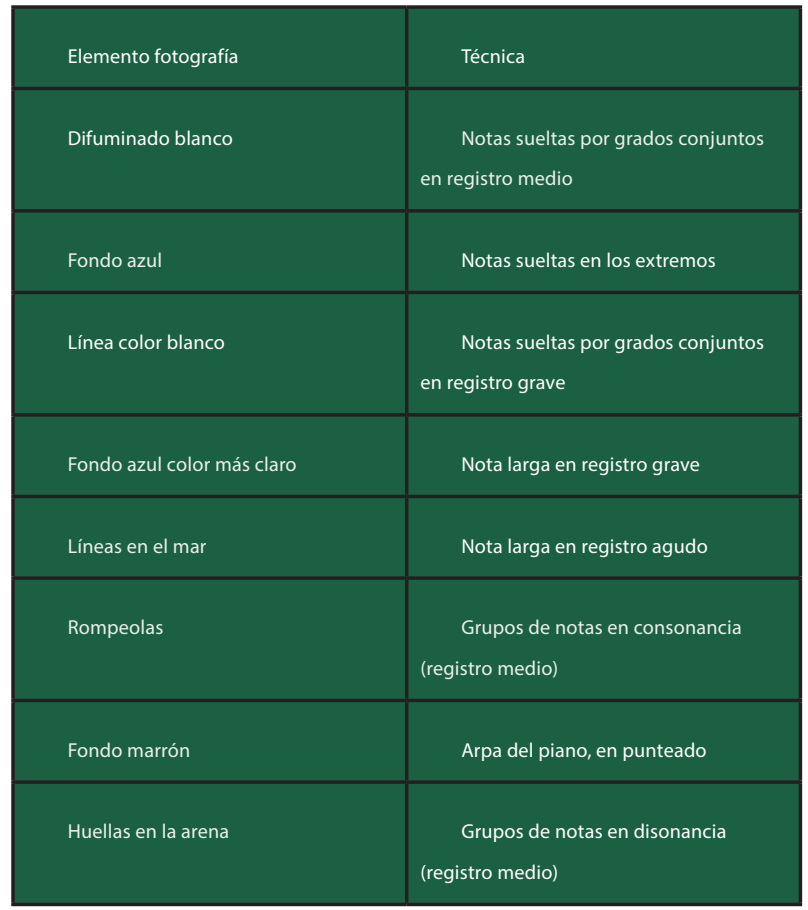

Nuevamente, la lectura de la fotografía para la interpretación pianística propongo que se haga igual que la lectura de un texto según cómo se hace en mi país.

\section{Fotografía 2}

\section{- Camino tradicional}

Continuando la idea de reflejar lo que cuenta la fotografía mediante la música, observé como aspecto más interesante el movimiento de las hojas de una palmera. Por ello, determiné que la composición sería nuevamente sencilla, con un ritmo ternario al estilo vals y en la tonalidad de La mayor, por ser el relativo mayor de Fa\# menor. Este último hecho, se ve nuevamente relacionado con las cuestiones comentadas en la fotografía 1 en relación mi 
asociación personal del mar con la luna. No obstante, es cierto que en este caso no hay presencia del mar pero creo que, socialmente, por su presencia en las playas e islas, la palmera mantiene una unión con la idea de playa y mar.

\section{- Camino de traducción}

En este caso, la búsqueda de sonidos se convierte en una tarea más complicada. Por lo tanto tomé dos decisiones: primero, la palmera sería mostrada sonoramente mediante el uso de las notas La\#, Do, Solb, Sol, Re\#, Si y Do, las cuales corresponden respectivamente a las letras $P, A, L, M, E, R$ y $A$ según el alfabeto comentado en la fotografía 1 en el apartado de este mismo camino. Dichas notas son realizadas en el registro grave del piano $y$, a su vez, se llevan a cabo notas ascendentes para la emisión de la idea altura. Finalmente, la segunda parte hace alusión al viento mediante glissandos ascendentes. El viento no es una idea propia de la fotografía pero, desde mi experiencia, es una forma de reflejar el movimiento de la palmera.

\section{- Camino de asociación}

Tras estudiar las formas y colores de la imagen, llegué a la conclusión que la lectura de la fotografía para la interpretación debía hacerse de abajo a arriba, debido a la forma de la palmera. Igualmente, dentro de la visualización de la fotografía-partitura, el cielo debía ser tomado como un constante. Basándome en estas ideas, determiné que la interpretación se debe iniciar mediante un glissando ${ }^{2}$ ascendente que tomara el mayor registro del piano, manteniéndose hasta el final con el uso del pedal izquierdo, siendo esto la lectura del cielo. A continuación, y según el estudio de formas realizado, se lleva a cabo una serie de cluster ${ }^{3}$ (consultar apartado 5, notas) ascendentes, los cuales serían el tronco.

Seguidamente, se realizan acordes en registro medio, los cuales son la copa de la palmera. Tras estos últimos acordes, se hace una bajada en glissando que hace alusión a la hoja de palmera descendente. Después, nos encontramos con tres hojas de palmera que no son ascendentes ni ascendentes, por lo que se hacen tres glissandos con subidas y bajadas pero en un registro medio para, después, llevar a cabo ocho glissandos con subidas y bajadas pero, en esta ocasión, con un registro más amplio, siendo de esta forma las ocho hojas visualizadas ascendentes.

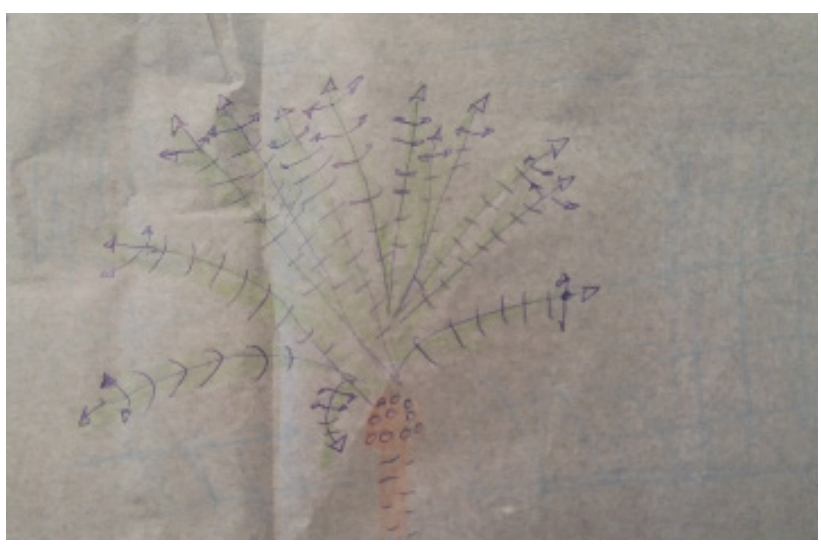

¿Qué veo en la imagen sonoramente?

- Camino de simbolización

Realizando nuevamente un análisis de la fotografía, concluí los siguientes elementos más destacables, ordenadas en orden de aparición descendente a ascendente: Fondo azul (constante), tronco, copa, hoja descendente, hojas que han empezado a descender y hojas ascendentes. Tras ello, asigné mediante el azar una de las técnicas mencionadas en la tabla del apartado 3.2. del presente artículo. Como resultado, quedó la siguiente simbolización de los elementos para la interpretación pianística: 


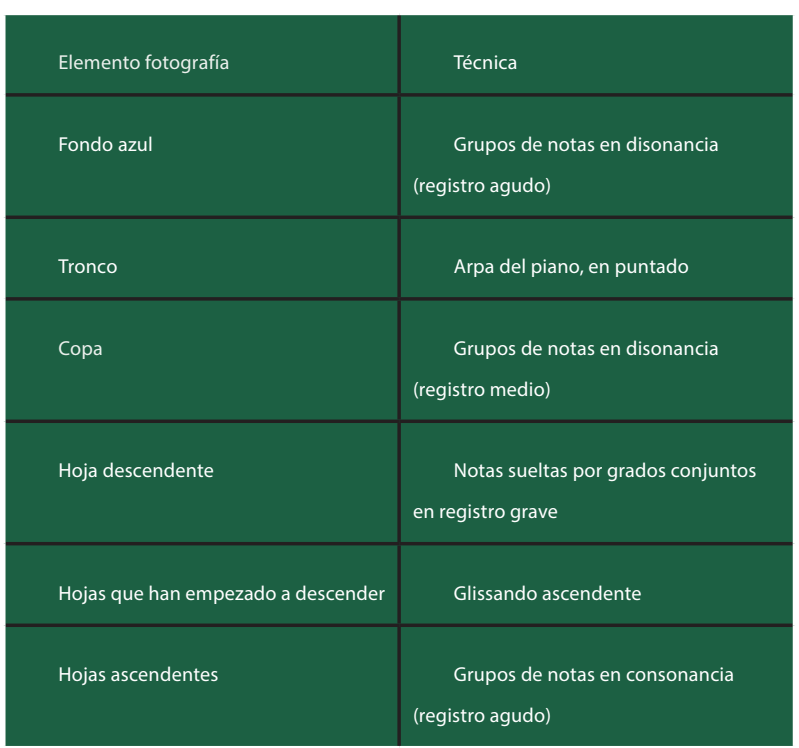

Al igual que en el caso anterior, la lectura de la fotografía para la interpretación pianística propongo que se haga de descendente a ascendente, quedando la simbolización del cielo siempre presente ${ }^{4}$.

\subsection{Resultados}

- Fotografía 1. Camino Tradicional: https://youtu.be/ taf7AtDq4mw

- Fotografía 1. Camino de Traducción: https://youtu.
be/E8ClcbULGaw

- Fotografía 1. Camino de Asociación: https://youtu. be/ukp2jR1NTOo

- Fotografía 1. Camino de Simbolización: https:// youtu.be/PGlahzrGcxo

- Fotografía 2. Camino Tradicional: https://youtu.be/ KvbarS5fbQw

- Fotografía 2. Camino de Traducción: https://youtu. be/o2pRsJqmqn8

- Fotografía 2. Camino de Asociación: https://youtu. $\mathrm{be} / \mathrm{gln} \_$E23z6Ck

- Fotografía 2. Camino de Simbolización: https:// youtu.be/W2t34b_U4PQ

4. Conclusiones del estudio

Tras la realización de la investigación artística presente en este artículo, concluyo que el uso de fotografías como herramientas para la interpretación musical mediante su uso como partitura, es un ejercicio interesante para el estudio personal de asociaciones de elementos visuales a aspectos sonoros. No obstante, cabe destacar que según la experiencia realizada, es una tarea complicada llevar a cabo esta propuesta sin estar impregnado/a de ideas socioculturalmente adquiridas. Este hecho sólo pude eliminarlo en el camino de simbolización dado que llevé a cabo una simbolización sonora de los elementos visuales mediante el azar. 
5. Notas

1.-¿Qué técnica de azar usé en el camino de simbolización?

La técnica utilizada es la misma que he empleado desde mi infancia y consiste en tomar papeles pequeños, asignándoles a cada uno de ellos uno de los aspectos que deben salir al azar. Se doblan los papeles y se arrojan juntos a un recipiente. El papel que se coge al azar, es el aspecto elegido.

2.-¿Qué es un glissando?

Un glissando (De pedro, 2001) es la pasada rápida por notas de forma ascendente o descendente, sin pensar en ninguna de manera determinada. El tiempo de realización es inexacto.

3.-¿Qué es un cluster?

En compositores actuales, como el caso de Ramón Roldán (según citado en García et al., s.f.) se suele simbolizar en las partituras mediante una línea vertical y se refiere a conjuntos de notas en grados conjuntos que deben ser tocadas a la vez.

4.-¿Cuál es mi abecedario musical?

Consiste en el desarrollo del abecedario europeo-español asignándole a cada letra, y en dicho orden, una nota partiendo desde el do central y en diferencia de cuarto de tono. Cuando finalice la existencia de sonidos por cuarto de tono, se vuelve al Do central y se hace la escala tonal mayor partiendo de la nota Do en doble sostenido $(\mathrm{x})$.

\section{Agradecimientos}

Gracias por acompañarme y ayudarme con tu ojo fotográfico Ali.

Gracias al profesor Fernando Hernández por relacionar mi comentario acerca de "sistematizar las emociones" con la notación musical.

Gracias a la profesora Lucía Loren por hacerme ver en el entorno recursos y obras estéticas 


\section{Referencias}

- Albéniz, I. (compositor) \& Lechner, L. (Ed.) (1996). Suite espagnole Opus 47. Piano. Alemania: Schott.

- Buj Corral, Marina (2013). Grafismos en la música: origen y desarrollo de las partituras gráficas. En Sinfonía Virtual, (24) 1-12. Recuperado de: http://www.sinfoniavirtual.com/revista/024/grafismos_musica_ partituras_graficas.pdf [Última visita: 31-1-2018].

- Cage, J. (2012). Silencio. Madrid: Árdora.

- Dobretzberger, F. [responsable del contenido] (s.f.). La octava cósmica: otros sonidos. En PlanetWare.de [En línea]. Disponible en: https://www.planetware.de/octava_cosmica/otros-sonidos.html [Última visita 9-22018].

- Espíritu Zalvaza, M. P. (2014). Aproximación a un proyecto de investigación en arte: "Navegar en el tiempo". En Terciocreciente, (5), 61-66. Recuperado de: http://www.terciocreciente.com/images/revistas/articulosn5/5-patricia-espiritu.pdf [Última visita 9-2-2018].

- Fubini, E. (2007). Capítulo 11: La música y la fusión de las artes. En La estética musical desde la Antigüedad hasta el siglo XX (313-266341).Madrid: Alianza Música.

- García Fernández, I. D. (2007). El grafismo musical en la frontera de los lenguajes artísticos. En Sinfonía Virtual, (5), 1-12. Recuperado de: http://www.sinfoniavirtual.com/revista/005/grafismo_musical_frontera_ lenguajes_artisticos.php [Última visita 9-2-2018].

- Gaspar, M. L. (2016, 4 de Febrero). La pianista Hélène Grimaud alerta sobre el agua en un disco "ecologista". En Agencia EFE [En línea]. Recuperado de: https://www.efe.com/efe/america/cultura/la-pianista-helenegrimaud-alerta-sobre-el-agua-en-un-disco-ecologista/20000009-2830291 [última visita 9-2-2018].

- Hernández Hernández, F. (2017). Apuntes de Sistematización y análisis del conocimiento artístico. En Máster de Investigación y Educación Estética: Artes, Música y Diseño [Material Inédito]. Jaén: Universidad de Jaén.

- May, J. (1993). The use of the Bach Motive in the Music of Arnold Schoenberg. En South African music studies: Samus; Matieland, 13, p. 43. Recuperado de: https://search.proquest.com/openview/0e01b3d6c7 f16fcb859bcf0cc56ab449/1?pq-origsite=gscholar\&cbl=2029870 [Última visita 9-2-2018].

- Ortega, J. F. (2008). Los sonidos del agua. En Revista Murciana de Antropología (15), 115-130. Recuperado de: http://revistas.um.es/rmu/article/view/108382 [Última visita 9-2-2018].

- Roldán Samiñán, R. (s.f.). Introducción a la música contemporánea. En García García, J. V.; Iglesias González, J.; Iglesias González, J.; Robles Ojeda, G. \& Roldán Samiñán, R. (s.f.). Lenguaje Musical Melódico VI. Grado Medio $2^{\circ}$ Curso, (99-129). Málaga: Ediciones Si bemol. 
- Bibliografía

- De Pedro, D. (2001). Teoría completa de la música (vol. 1 y 2). Madrid: Real Musical.

- Vídeos de interés:

- Haubenstock-Ramati, Roman [Jimbo, canal youtube] (2011, 14 de mayo). Alone I (1965) Roman Haubenstock-Ramati (1919-94). En youtube.es [En línea]. Recuperado de: https://www.youtube. com/watch?v=WpbxxbQcclY

- Xenakis, Innais [Pierre Carré, canal youtube] (2017, 30 de abril). Innais Xenakis - Pithoprakta (w/ graphical score) [Grabado por Tamayo, A., (Dir. orquesta). En youtube.es [En línea]. Recuperado de: https://www.youtube.com/watch?v=nvH2KYYJg-o

- Plourde, Michel [Michel Plourde, canal youtube] (2017, 27 de junio). How to read \& Write Graphic Notation (part 2) - Music Stuff With Spock \#9. En youtube.es [En línea]. Recuperado de: https:// www.youtube.com/watch?v=LreDyyOi1yE 